\title{
Dynamic Response of Membrane Structures Under Water Impact
}

\author{
Li Yantao", Xu Xiaochen ${ }^{2}$, Xu Zhihong ${ }^{1, ~ * ~}$ \\ ${ }^{1}$ School of Science, Nanjing University of Science and Technolgy, Nanjing, China \\ ${ }^{2}$ China Nanjing Institute of Electronic Technology, Nanjing, China
}

\section{Email address:}

1308916219@qq.com (Li Yantao), xuzh@njust.edu.cn (Xu Zhihong)

${ }^{*}$ Corresponding author

\section{To cite this article:}

Li Yantao, Xu Xiaochen, Xu Zhihong. Dynamic Response of Membrane Structures under Water Impact. Science Discovery. Vol. 8, No. 5, 2020, pp. 90-96. doi: 10.11648/j.sd.20200805.13

Received: August 24, 2020; Accepted: September 19, 2020; Published: September 23, 2020

\begin{abstract}
In this paper, the dynamic response of ship-borne membrane radome under the impact of water was investigated.Based on smooth particle hydrodynamics (Smooth particle hydrodynamics SPH), a fluid-structure coupling model was established, to simulate the membrane structure impacted by water, and the dynamics of the shipboard radome membrane structure under the impact of the breaking wave water body are simulated by the computer numerical simulation method response.The influence of the initial velocity of water, the angle of incidence, the initial pretension of the membrane structure and the tensile shape of the membrane surface on the maximum impact deformation and maximum impact stress of the membrane surface are analyzed. According to experimental analysis, under the same impact, the film structure with greater pretension has stronger resistance to impact, that is to say, under the same impact of water body, the greater the pretension of the membrane structure, the smaller the maximum internal stress change amplitude when it is impacted. Therefore, a certain optimal prestress is found to make the entire radome membrane structure suffer the same impact. The minimum maximum membrane stress is generated at the time, so as to achieve the purpose of protecting the fabric membrane structure from large stress and deformation; at the same impact speed, the vertical impact angle has the greatest impact on the membrane structure; under the same impact and pretension, the saddle-shaped film surface has stronger resistance to impact than the flat film surface.
\end{abstract}

Keywords: Wave Load, Orthotropic Membrane, Smooth Particle Hydrodynamics, Fluid-solid Coupling, Nonlinear Materials, Simulation

\section{膜结构在水体冲击下的动力响应}

\author{
李言涛 ${ }^{1}$, 徐晓晨 ${ }^{2}$, 徐志洪 ${ }^{*}$ \\ 1南京理工大学理学院, 南京市, 中国 \\ 2南京电子技术研究所, 南京市, 中国 \\ 邮箱 \\ 1308916219@qq.com（李言涛）, xuzh@njust.edu.cn（徐志洪）
}

摘要: 研究了舰载膜结构雷达罩在水体冲击作用下的动力响应问题。采用光滑粒子流体动力学方法 (Smooth particle hydrodynamics, SPH) 建立了水体冲击薄膜的流固耦合数值计算模型, 通过计算机数值仿真的方法模拟了舰载雷达罩 膜结构在碎浪水体荷载冲击下的动力响应。主要分析了水体的初始速度、入射角度、膜材结构的初始预张力以及膜面 张拉形状因素对膜面的最大冲击变形和最大冲击应力的影响规律。并由实验分析得到, 在相同冲击作用下, 预张力越 大的膜结构抵抗冲击的能力越强, 即在相同的水体冲击作用下, 预张力越大的膜结构, 其遭受冲击时产生的最大内应 力变化幅度越小, 因此找到某一最佳预应力, 使整个雷达罩膜结构在遭受相同冲击时产生最小的最大膜内应力, 从而 
达到保护织物膜结构不产生较大应力和形变的目的; 在相同的冲击速度下, 垂直的冲击角度对膜结构造成的影响最大; 在相同冲击作用和预张力的情况下，马鞍形膜面比平面膜面抵抗冲击的能力强。

关键词: 浪荷载, 正交各向异性薄膜, 光滑粒子流体动力学, 流固耦合, 非线性材料, 仿真

\section{1. 引言}

膜结构雷达罩作为一种新型的雷达保护结构, 具有重 量轻、覆盖面积大、透波效果好等特点, 适用于舰载远程 探测雷达。在舰船高速行驶时产生的浪花形成的水体冲击 荷载作用在膜结构的雷达罩, 产生过大的形变会对雷达天 线工作性能产生影响, 因此对雷达罩在浪荷载冲击下的动 力响应研究就显得尤为重要。

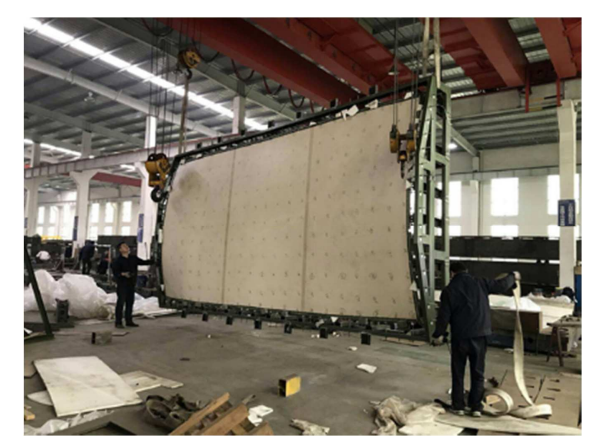

图1 马鞍形雷达罩膜结构。

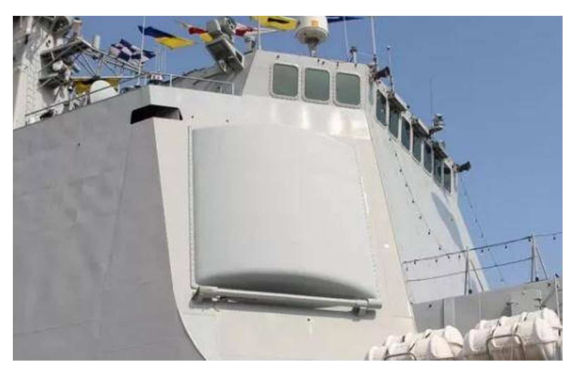

图2 相控阵圆弧形雷达罩膜结构。

膜结构的动力响应是目前膜结构研究的一个热点, 刘 长江 [1-2]等人分别研究了正交各向异性鞍形膜在非线性 风载作用下的流固耦合现象和稳定性, 并采用和有限元数 值模拟研究了四边固定预拉伸正交各向异性织物在动力 冲击下的非线性振动。李董[3]等人从理论上和实验上研究 了矩形预应力膜在均匀冲击载荷作用下的动力响应。杭石 [4]等人也对大空间膜的非线性振动和大变形做了研究。 Mélanie Delapierre[5]等人研究了均值环形膜材料在均匀 横向荷载下的非线性振动。Ali Asghar Atai[6]等人通过力 学方法对各向异性膜材料进行了数值分析, 但是前人对膜 结构在水体冲击下的流固耦合的动力响应研究的较少。

本文研究了膜结构在水体冲击下的流固耦合动力响 应，分析了不同水体初始速度、不同水体冲击角度、不同 膜预张力以及不同膜面结构形式下的薄膜动力响应以及 最大形变, 为膜结构舰载雷达罩的设计提供理论依据。

\section{2. 数值计算原理及模型描述}

\section{1. 光滑粒子流体动力学法简述}

光滑粒子流体动力学法 ( smooth particle hydrodynamics, SPH） [7-15]是模拟流体流动的一种拉格 朗日型粒子方法, 它无需网格对计算领域进行划分, 而是 将计算域离散为一系列有相互作用的粒子[16]。

传统 SPH方程的构造分为核函数插值和粒子近似两 个关键步骤, 分别实现了场变量或场变量梯度的插值和对 核函数估计积分表达式的粒子离散[17]。

1) 核函数插值

对于一个连续的光滑函数 $\mathrm{f}(\mathrm{r})$, 在定义域 $\Omega$ 上一点的 函数值可表示为

$$
\mathrm{f}(r)=\int_{\Omega} f\left(r^{\prime}\right) \delta\left(r-r^{\prime}\right) d r^{\prime}
$$

其中, $\mathrm{r}$ 为空间矢量位置; $\delta\left(r-r^{\prime}\right)$ 为狄拉克 $\delta$ 函数, 实际中狄拉克 $\delta$ 函数难以实现，在数值计算中用光滑函数 $\mathrm{W}\left(\mathrm{r}-r^{\prime}, \mathrm{h}\right)$ 来取代 $\delta$ 函数, 则 $\mathrm{f}(\mathrm{r})$ 可近似为:

$$
\langle f(r)\rangle=\int f\left(r^{\prime}\right) W\left(r-r^{\prime}, h\right) d r^{\prime}
$$

$\mathrm{W}\left(r-r^{\prime}, h\right)$ 即为 $\mathrm{SPH}$ 方法中的核函数, 其值取决于两 点之间的距离 $\left|r-r^{\prime}\right|$ 和光滑长度 $\mathrm{h}$, 它与光滑因子 $\mathrm{k}$ 一同决 定了光滑核函数影响域的大小。

2) 粒子近似

对于某个求解域, 用粒子的体积 $V_{j}$ 来近似积分粒子 $\mathrm{j}$ 处的无穷小微元 $\mathrm{d} r^{\prime}$, 则粒子的质量 $m_{j}$ 可表示为

$$
m_{j}=\Delta V_{j} \rho_{j}
$$

其中, $\rho_{j}$ 为粒子 $\mathrm{j}$ 的密度 $(\mathrm{j}=1,2, \cdots, \mathrm{N}), \mathrm{N}$ 为紧支域内 的粒子总数。

对(2)式进行粒子离散化, 得

$$
\begin{gathered}
\langle f(r)\rangle=\int_{\Omega} f\left(r^{\prime}\right) W\left(r-r^{\prime}, h\right) d r^{\prime} \\
\approx \sum_{j=1}^{N} f\left(r_{j}\right) W\left(r-r_{j}, h\right) \Delta V_{j} \\
=\sum_{j=1}^{N} f\left(r_{j}\right) W\left(r-r_{j}, h\right) \frac{m_{j}}{\rho_{j}}
\end{gathered}
$$

对粒子 $\mathrm{i}$ 处场函数的粒子估计最终为

$$
\left\langle f\left(r_{i}\right)\right\rangle=\sum_{j=1}^{N} \frac{m_{j}}{\rho_{j}} f\left(r_{j}\right) W_{i j}
$$

其中,

$$
W_{i j}=W\left(r_{i}-r_{j}, h\right)
$$


式(5)表明，粒子 $\mathrm{i}$ 处的场函数值可以通过核函数对该 粒子紧支域内所有粒子的函数值加权平均得到。

\section{2. 雷达罩模型}

雷达罩膜材料为聚四氟乙烯(Poly tetra fluoroethylene, PTFE) 建筑用膜, 膜材形状为 $6 \mathrm{~m} \times 9 \mathrm{~m}$ 厚度为 $1.2 \mathrm{~mm}$ 的矩形, 采用预张力的形式固定在矩形框架上形成预应力张拉膜 结构。如图3所示,

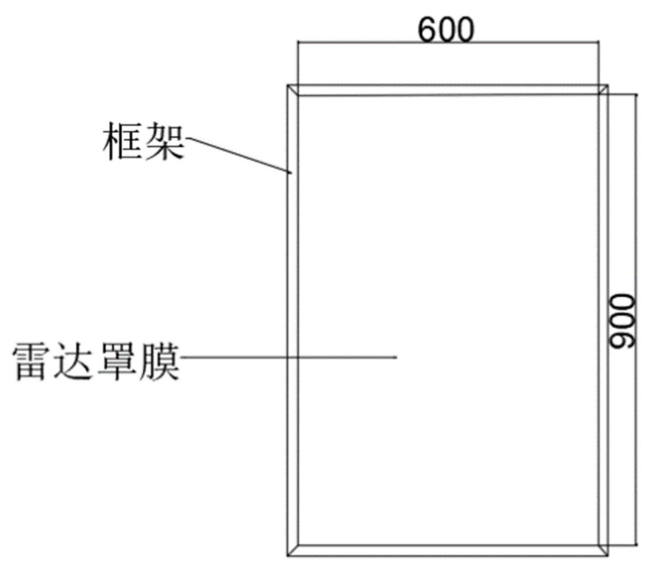

图3 雷达罩膜结构示意简图。

数值仿真中忽略雷达罩框架的变形, 近似为预张力正 交各向异性薄膜。雷达罩采用shell壳体模型，使用elastic 中Engineering Contants属性材料, 并将长边方向定义为材 料主方向，雷达罩材料的各项参数定义如下

表1 膜各项参数。

\begin{tabular}{lllll}
\hline 名称 & $\boldsymbol{\rho}_{\mathbf{0}} /\left(\boldsymbol{k g} / \boldsymbol{m}^{\mathbf{3}}\right.$ & $\boldsymbol{E}_{\boldsymbol{x}} /(\boldsymbol{M p a})$ & $\boldsymbol{E}_{\boldsymbol{y}} /(\boldsymbol{M p a})$ & $\boldsymbol{E}_{\boldsymbol{z}} /(\boldsymbol{M p a})$ \\
\hline 膜 & 1700 & 1400 & 900 & 1150 \\
& $\boldsymbol{G}_{\boldsymbol{x}} /(\boldsymbol{M p a})$ & $\boldsymbol{G}_{\boldsymbol{y}} /(\boldsymbol{M p a})$ & $\boldsymbol{G}_{\boldsymbol{z}} /(\boldsymbol{M p} \boldsymbol{p})$ & $\mathbf{p}$ \\
& 28 & 18 & 23 & 0.3 \\
\hline
\end{tabular}

\section{3. 水体模型}

水体形状为液滴形, 采用 $\mathrm{sph}$ 方法建模并定义为材料 模型EOS方程US-UP类型的流体材料, 并在Eos中设置 Us-UpHugoniot[18]方程。

$$
P_{H}=\frac{\rho_{0} c_{0}^{2} \eta}{(1-s \eta)^{2}}
$$

其中 $\mathrm{c} 0$ 为 $U s-U p$ 曲线的截距, $\mathrm{s}$ 是 $U s-U p$ 曲线斜率的系 数, Gamma0 是Gruneisen gamma, Us应力波传播速度, Up 质点运动速度。利用 $\mathrm{SPH}$ 方法将每个水粒子特征尺寸定义 为 $5 \mathrm{~mm}$, 并赋予材料 $\mathrm{PC} 3 \mathrm{D}$ 类型的单元族。

表2 水各项参数。

\begin{tabular}{lllll}
\hline 名称 & $\boldsymbol{\rho}_{\mathbf{0}} /\left(\boldsymbol{k g} / \boldsymbol{m}^{\mathbf{3}}\right)$ & $\boldsymbol{C}_{\mathbf{0}} /(\boldsymbol{m} / \boldsymbol{s})$ & $\mathbf{S}$ & $\mathbf{G a m m a 0}$ \\
\hline 水 & 1000 & 1400 & 0 & 0 \\
\hline
\end{tabular}

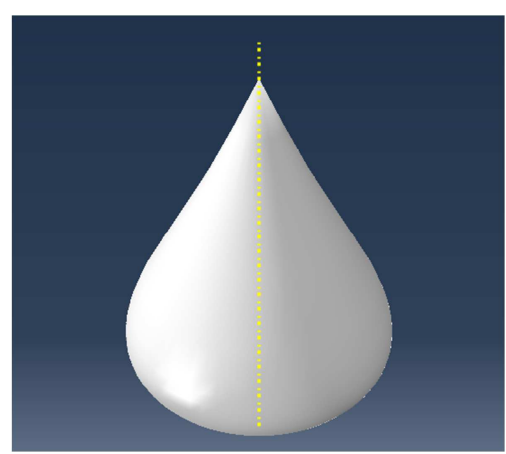

图4 浪荷载近似水体模型示意图。

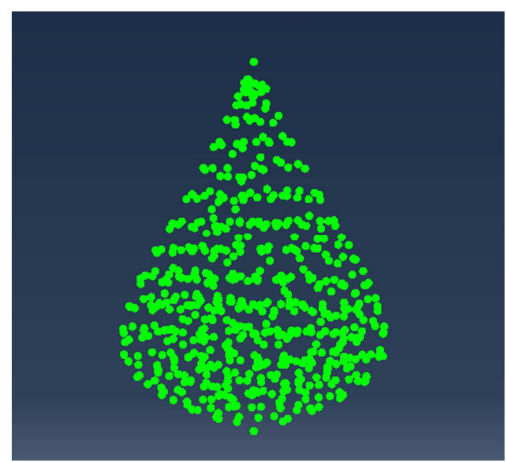

图5 水体光滑粒子划分模型图。

\section{4. 几何模型以及边界条件}

使用上述方法所建立的水体和雷达罩模型, 建立如图 所示的几何模型。其中水体近似为上下两圆雉相结合的近 似液滴形模型, 上部近似高度为 $200 \mathrm{~mm}$, 下部近似高度为 $50 \mathrm{~mm}$, 近似半径为 $100 \mathrm{~mm}$, 近似体积为 $2.62 \times 106 \mathrm{~mm}^{3}$, 质量为 $2.62 \mathrm{~kg}$, 光滑粒子直径为 $5 \mathrm{~mm}$, 约有 20960 个光滑 粒子。雷达罩平面模型采用 $900 \mathrm{~mm} \times 600 \mathrm{~mm} \times 1.2 \mathrm{~mm}$ 的壳体 模型, 马鞍形模型采用原 $900 \mathrm{~mm} \times 600 \mathrm{~mm} \times 1.2 \mathrm{~mm}$, 弧顶高 度为 $60 \mathrm{~mm}$ 的圆弧形膜结构通过施加预应力形成, 马鞍形 膜结构最终形状与膜内初始应力相关。水体的到雷达罩的 初始距离 $60 \mathrm{~mm}$, 薄膜采用四边固定的固定方式, 并施加 预应力场, 水体冲击位置为薄膜中心, 水体粒子与膜表面 无摩擦。

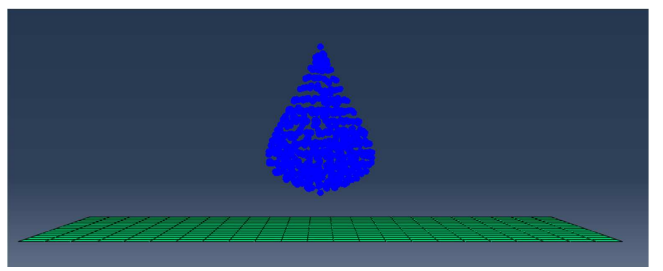

图6 水体冲击平面膜结构数值计算模型图。

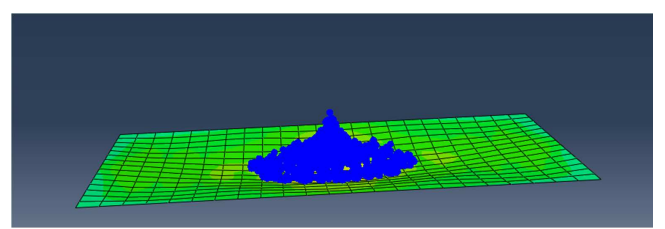




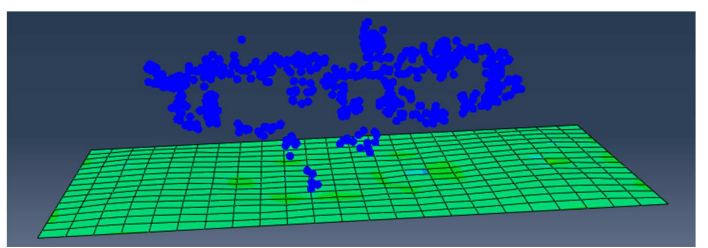

图7 水体冲击平面膜结构数值计算仿真过程图。

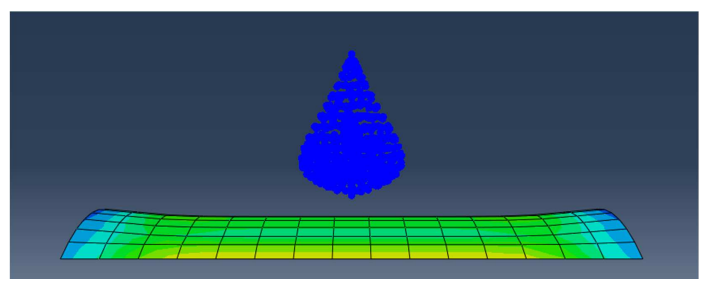

图8 水体冲击马鞍形膜结构数值计算模型图。
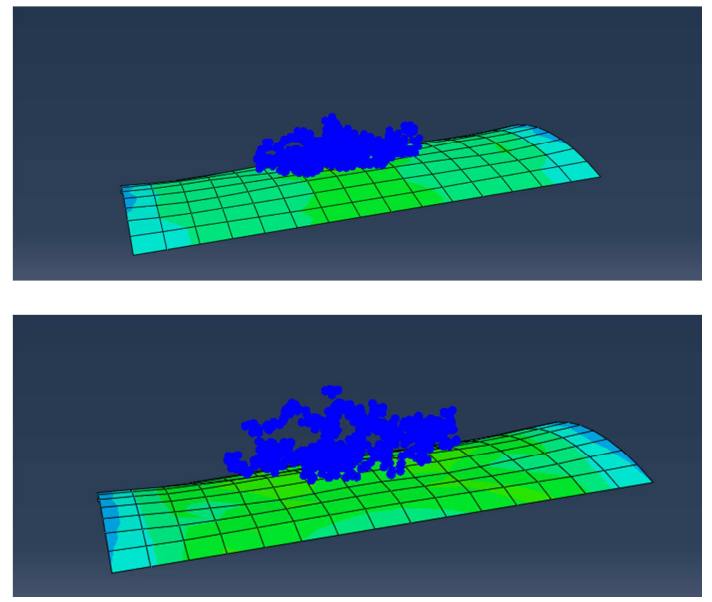

图9 水体冲击马鞍形膜结构数值计算模型图。

\section{3. 仿真计算结果及分析}

通过Abaqus进行水体冲击膜结构的分析计算后, 得到 膜结构的最大位移以及最大面内应力。图10、图11所示为 膜中初始预应力为 $10 \mathrm{MPa}$, 水体冲击速度为 $20 \mathrm{~m} / \mathrm{s}$, 垂直 入射时最大变形点的变形和面内应力的时程曲线

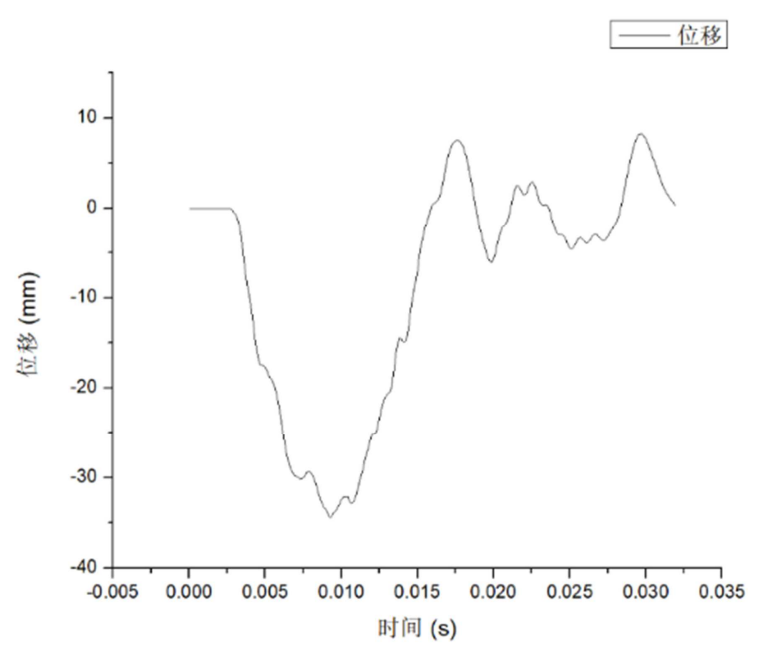

图10 膜结构变形最大位置点位移曲线图。

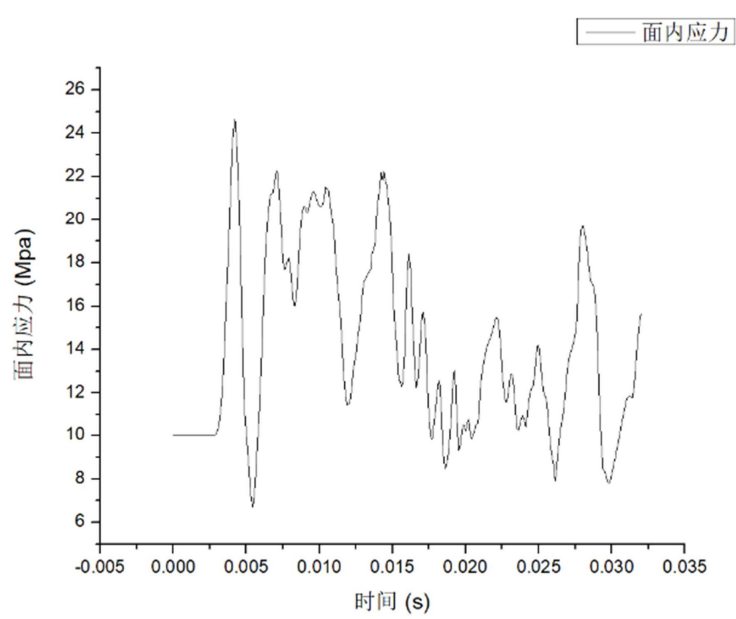

图11 膜结构最大面内应力曲线图。

\section{1. 水体初始速度对膜结构最大面内应力及最大变形影} 响

设置初始膜内应力为 $10 \mathrm{Mpa}$, 分别选用 $10 \mathrm{~m} / \mathrm{s}, 15 \mathrm{~m} / \mathrm{s}$, $20 \mathrm{~m} / \mathrm{s} ， 25 \mathrm{~m} / \mathrm{s} ， 30 \mathrm{~m} / \mathrm{s} ， 35 \mathrm{~m} / \mathrm{s} ， 40 \mathrm{~m} / \mathrm{s}$ 这7种水体入射速度 冲击薄膜, 得到薄膜的最大位移变形和最大膜面内应力的 变化。

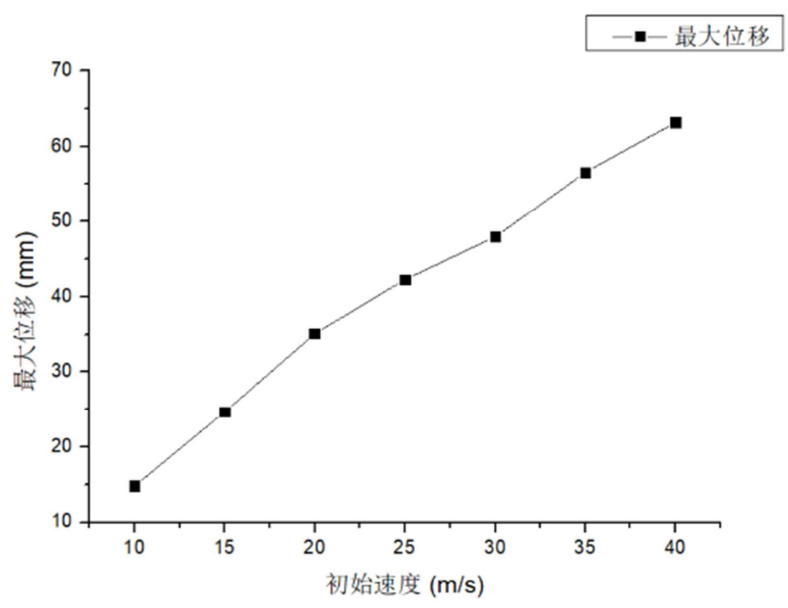

图12 不同初始速度膜结构最大位移图。

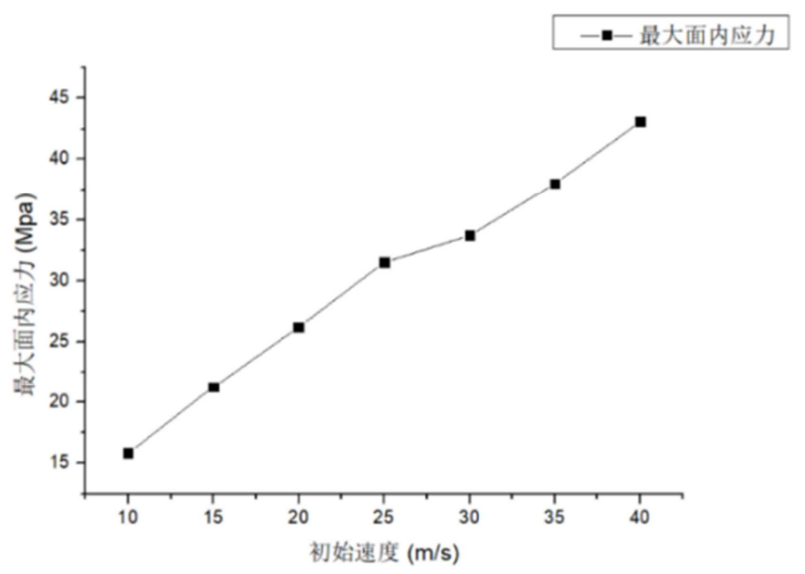

图13 不同初始速度膜结构最大面内应力图。 
从上图分析可知, 在相同初始膜内应力改变水体入射 速度的条件下, 膜结构的最大位移随着水体入射速度的增 加而增大, 呈现一直上升不存在平台和断层的情况; 膜结 构的最大面内应力随水体入射速度的增加而增加。

\section{2. 膜结构预张力对膜结构最大面内应力及最大变形影}

\section{响}

设置水体初始入射速度为 $20 \mathrm{~m} / \mathrm{s}$, 通过改变膜内初始 预应力场, 分别选用 $0 \mathrm{Mpa}, 10 \mathrm{Mpa}, 15 \mathrm{Mpa}, 20 \mathrm{Mpa}, 25 \mathrm{Mpa}$, $30 \mathrm{Mpa}$ 的初始预应力场, 得到薄膜的最大位移变形和最大 膜面内应力的变化, 并计算膜结构最大膜面内应力与初始 膜面内应力差。

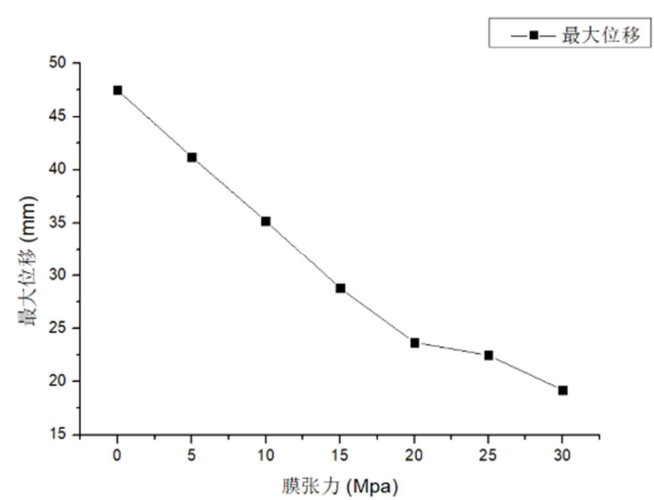

图14 不同预应力下膜结构最大位移图。

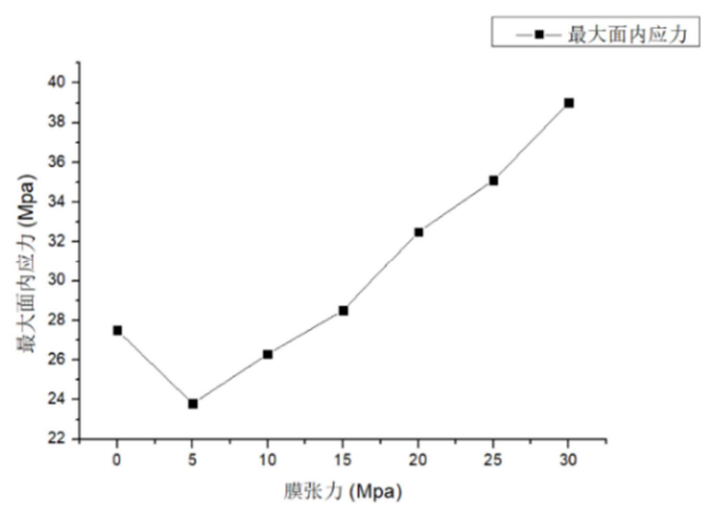

图15 不同预应力下膜结构最大面内应力图。

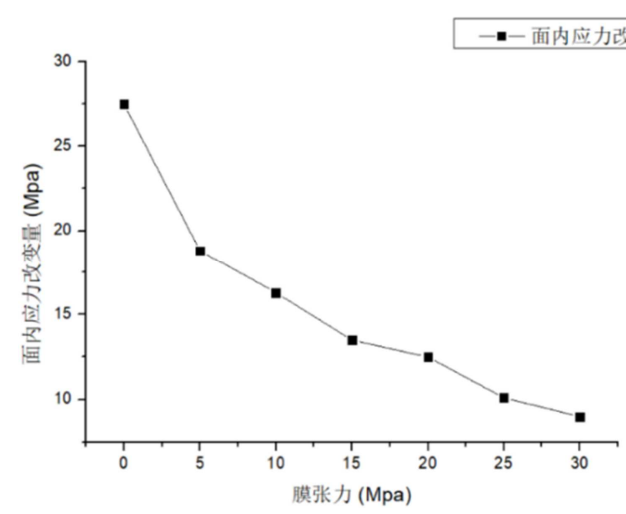

图16 不同预应力下膜结构最大面内应力增加图。
在相同初始入射速度改变初始膜结构内应力的条件下, 随着膜内初始应力的增加, 其抵抗水冲击的能力也相应提高, 膜结构的最大位移逐渐变小。当膜结构的初始应力达到 $20 \mathrm{Mpa}$ 之后, 对位移的减小作用逐渐减小, 在 $20 \mathrm{Mpa}$ 左右时 膜结构的最大位移已经降低到 $24 \mathrm{~mm}$, 继续增加膜结构内应 力对最大位移的减少作用不是很明显, 反而会持续增大冲击 后膜内最大应力, 使结构的可靠度降低, 产生破坏的危险。

但是从膜内最大应力来看, 初始膜预应力能够明显降 低仿真膜受到水体冲击后的最大膜内应力, 并且能在一定 程度上减少仿真膜的最大位移。值得注意的是, 增加膜结 构内初始应力能够有效降低仿真膜受到水体冲击后的最 大位移, 增加膜结构内初始应力能够有效降低膜内最大应 力的增加量, 即膜内最大应力的增加量随初始膜结构预应 力的增加而减少。

\section{3. 水体入射角度对膜结构最大面内应力及最大变形影} 响

水体初始入射速度为 $20 \mathrm{~m} / \mathrm{s}$, 薄膜面内预应力场为 $10 \mathrm{Mpa}$, 水体分别选用垂直膜面, 与膜面法线成 15 度夹角, 与膜面法线呈 30 度夹角, 与膜面法线成 45 度夹角四种入射 角度, 来模拟现实情况下雷达罩膜承受来自不同方向角度 的浪荷载冲击, 得到薄膜的最大位移变形和最大膜面内应 力的变化曲线。

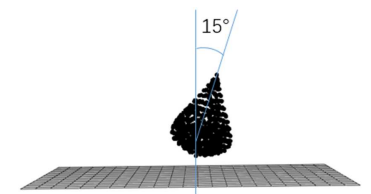

图17 与薄膜法线成 15 度冲击膜结构仿真示意图。

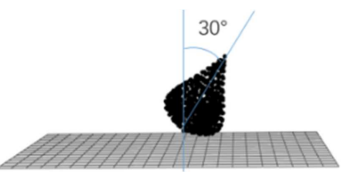

图18 与薄膜法线成30度冲击膜结构仿真示意图。

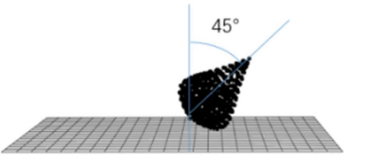

图19 与薄膜法线成 45 度冲击膜结构仿真示意图。

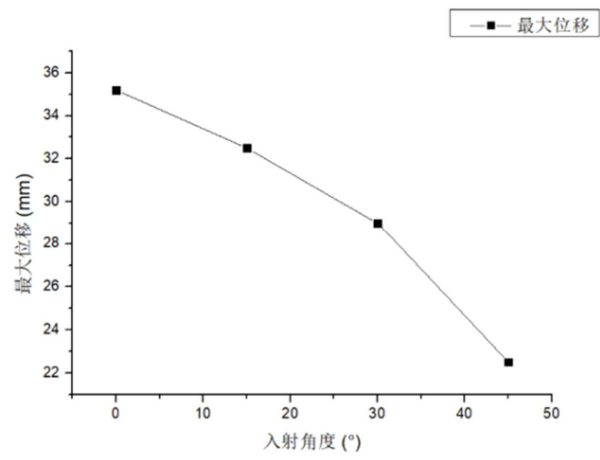

图20 不同冲击角度下膜结构最大位移图。 


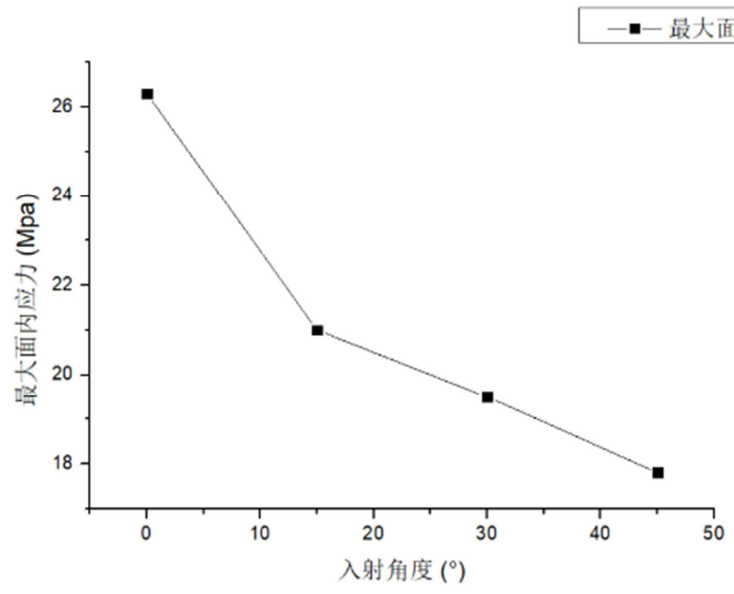

图21 不同冲击角度膜结构最大面内应力图。

在保持相同入射速度和膜结构内初始应力的条件下， 水体入射与膜面法线之间的夹角越大, 产生的冲击力越小, 相应的最大位移和最大面内应力也减小, 但是两者的减小 方式有所差别。入射角度越大, 最大位移的减小量越大, 最大位移的减小越明显; 入射角度越大, 膜内的最大应力 的减少量几乎不变, 几乎是以直线的形式均匀下降的。

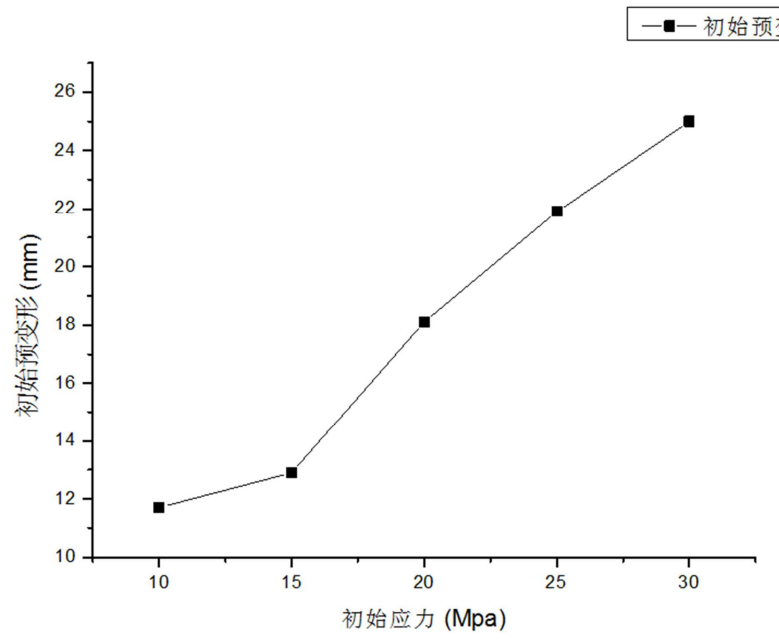

图22 不同预张力马鞍形膜结构初始位移。

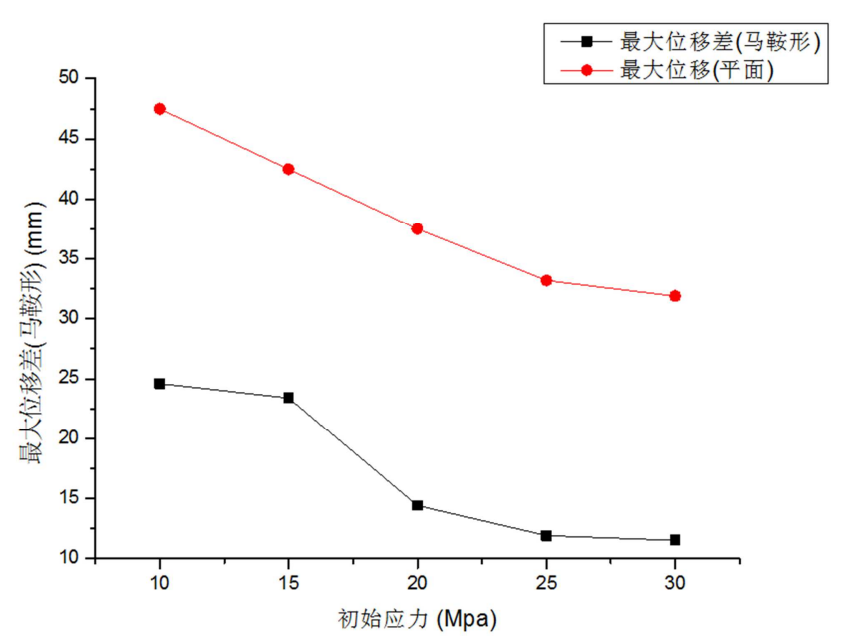

图23 不同预张力膜结构最大位移图。

\section{4. 膜面结构形式对膜结构最大面内应力及最大变形影 响}

由于马鞍形结构的雷达罩膜的最终形状受到初始预 应力的影响(初始预应力越大, 膜结构中心位置下降幅度 越大), 因此马鞍形膜结构的最大变形需要减去先前应预 应力所预先变形的部分。仿真实验中预先设置水体冲击速 度均为 $30 \mathrm{~m} / \mathrm{s}$, 通过改变两种类型膜结构的初始预张力进 行控制变量仿真实验。

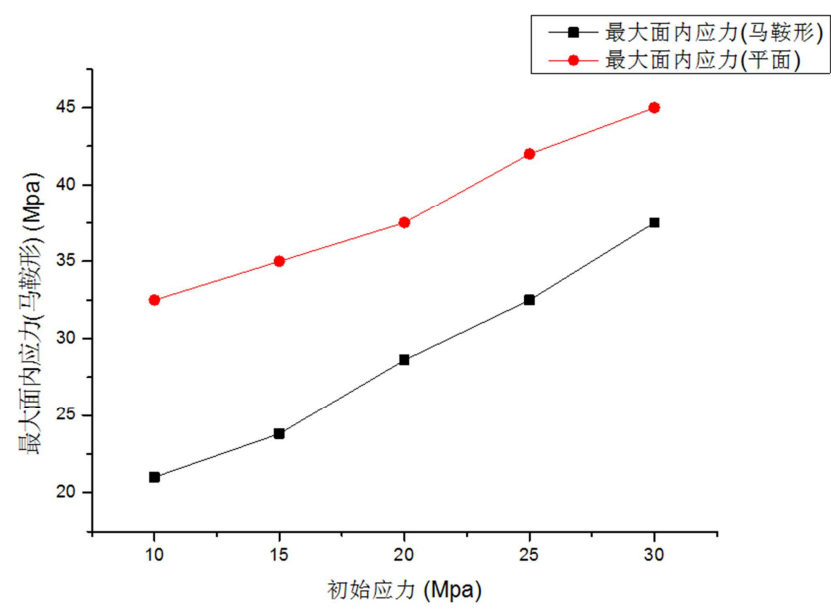

图24 不同预张力膜结构最大面内应力。

通过给定两种膜结构 $10 \mathrm{Mpa}$ 的初始预张力, 改变水体 的冲击速度, 得到以下关于两种膜结构面内最大应力和面 外最大位移的变化图。

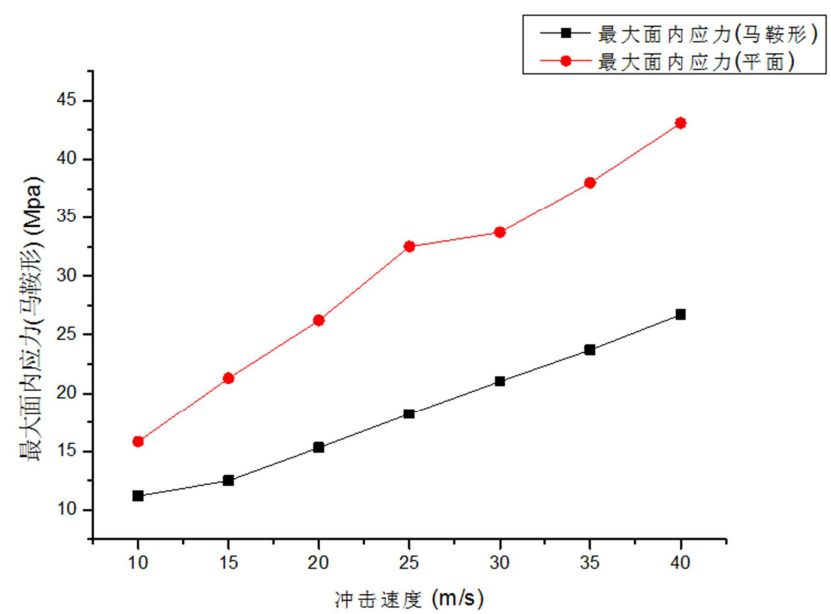

图25 不同冲击速度膜结构最大面内应力。

采用通过在不同初始预应力和不同水体冲击速度的 试验数据可以明显看出, 马鞍形膜结构不论是从膜结构的 稳定性还是膜结构内应力的变化幅度上都更优于平面膜 结构, 并且在减少雷达罩膜结构形变和面内应力变化上具 有非常大的优势, 膜面中心平均最大位移量和最大面内应 力均是是平面膜结构的二分之一左右, 而膜内应力变化幅 度也只有平面膜的二分之一。 


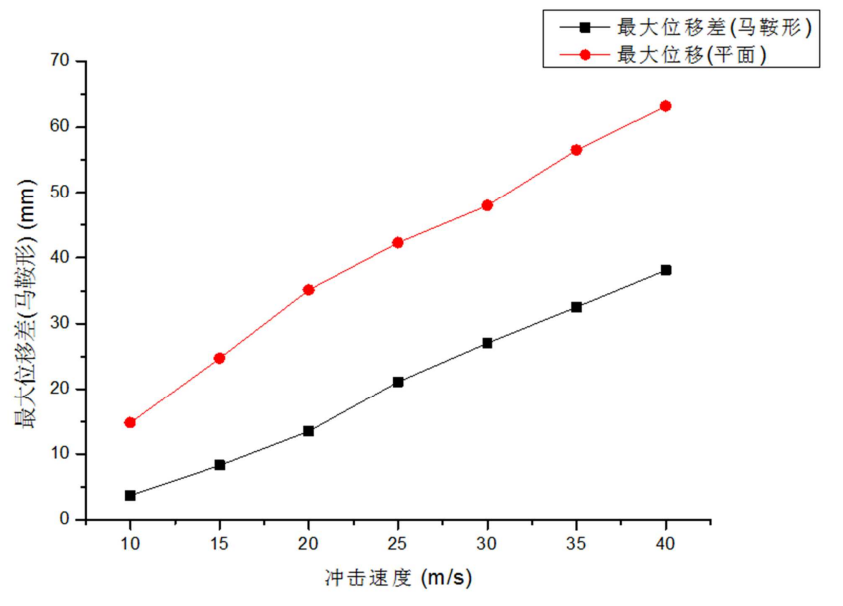

图26 不同冲击速度膜结构最大位移。

\section{4. 结论}

采用数值模拟的方法对水体对舰载雷达罩膜结构的 冲击进行了数值模拟计算, 应用Abaqus软件以及 SPH分析 方法对模型进行了分析计算, 从对实验的数据分析以及结 合膜结构的自身性质后能够得到以下几点结论:

1) 本文使用SPH法对正交各项异性材料的流固耦合 动力学冲击进行模拟和分析, 提供了一种建模思路;

2) 增加膜结构内初始应力可以明显改善其抗水冲击 的能力, 膜结构内初始应力越大, 其抵抗变形的能力越强, 受到相同冲击其最大膜内应力的增加量也越小，但是其初 始膜内应力会增大冲击后膜内最大应力, 因此在实际工程 中要根据膜材的破坏极限和雷达罩要求的允许最大变形 来确定其初始预应力;

3) 水体垂直冲击膜面时产生的面内应力和最大位移 相较与其他角度入射都要大, 所以在确定膜材变形和应力 可靠度时优先考虑水体垂直入射的情况比较节约时间和 计算成本。

4 ）无论是从最大膜面内应力还是从最大面外位移来 看, 马鞍形膜结构的稳定性均强于平面形膜结构。抛开马 鞍形膜结构本身的制作难度和成本, 马鞍形雷达罩膜结构 形式在实际应用中在各方面都是优于平面膜结构。

\section{参考文献}

[1] Liu Changiiang, Deng Xiaowei, Liu Jian, et al. Impact-induced nonlinear damped vibration of fabricmembrane structure: Theory, analysis, experiment and parametric study [J]. 2019.

[2] Liu Changjiang, Deng Xiaowei, Zheng Zhoulian. Nonlinearwind-induced aerodynamic stability of orthotropic saddle membrane structures [J]. Journal of Wind Engineering and Industrial Aerodynamics, 2017, 164:119-127.
[3] Li Dong, Zheng Zhoulian, Liu Caoyu, et al. Dynamic response of rectangular prestressed membrane subjected to uniform impact load [J]. Archives of Civil and Mechanical Engineering, 2017, 17(3):586-598.

[4] Hang Shia, Chao Wangb, et al. An active controlstrategy to suppress nonlinear vibrations of large spacemembranes[J]. Acta Astronautica 155 (2019) 80-89.

[5] Delapierre, Mélanie, Lohaus SH, ellegrino S. Nonlinear vibration of transversely-loaded spinning membranes [J]. Journal of Sound and Vibration, 2018, 427:41-62.

[6] Atai A, Steigmann D J. Numerical analysis of wrinkled, anisotropic, nonlinearly elastic membranes [J]. Mechanics Research Communications, 2014, 57:1-5.

[7] Ambroziak, Andrzej. Mechanical properties of Precontraint $1202 \mathrm{~S}$ coated fabric under biaxial tensile test with different load ratios [J]. Construction and Building Materials, 2015, 80:210-224.

[8] 李否, 马宏吴, 沈兆武等. 聚能射流侵彻钢靶的SPH-FEM数值 模拟[J].南京理工大学学报,2013,37(02):226-232。

[9] Zheng Hana, Bin Sua, Yange Lia, et al. Numerical simulation of debris-flow behavior based on the SPH method incorporating the Herschel-Bulkley-Papanastasiou rheology model [J]. Engineering Geology, 255(2019)26-36.

[10] Xiangwei Dong, Xiaoping Huang, Jianlin Liua. Modeling and simulation of droplet impact onelasticbeams based on SPH [J]. European Journal ofMechanics/ASolids, 75(2019)237-257.

[11] 李世杰,王艾伦,刘向军等.基于SPH算法土壤水射流冲击演 化数值仿真研究 [J].计算机仿真,2019,36(03):243-247+384。

[12] Huabin Shi, Pengfei Si, Ping Dong, Xiping Yu. A two-phase SPH model for massive sediment motion in free surface flows [J]. Advances in Water Resources, 2019, 129.

[13] 周楠,王金相,谢君等.两种数值算法在球形弹丸侵彻复合靶 中的应用 [J].南京理工大学学报,2014,38(02):210-215+221。

[14] Lu Wanga, Fei Xua, Yang Yanga. SPH scheme for simulating the water entry of an elastomer[J]. Ocean Engineering, 178(2019)233-245.

[15] 赵晓宁,何勇, 张先锋等. A3 钢抗高速杆弹侵彻的数值模拟与 实验研究[J].南京理工大学学报,2011,35(02):164-167。

[16] 刘桂荣. 光滑粒子流体动力学 $[M]$. 湖南大学出版社, 2005 .

[17] J K Chen, J E Beraun, C J Jih. An improvement for tensile insta-bility in smoothed particle hydrodynamics [J]. Computational Me-chanics, 1999, 23: 279-287.

[18] Mausbach P, May H O. Direct molecular simulation of the Grüneisen parameter and density scaling exponent in fluid systems[J]. Fluid Phase Equilibria, 2014, 366:108-116. 